\title{
Optimality Conditions for Nondifferentiable Multiobjective Semi-Infinite Programming Problems
}

\author{
D. Barilla, G. Caristi, and A. Puglisi \\ Department of Economics, University of Messina, Via dei Verdi 75, Messina, Italy \\ Correspondence should be addressed to G. Caristi; gcaristi@unime.it
}

Received 2 May 2016; Accepted 18 August 2016

Academic Editor: Jozef Banas

Copyright ( 2016 D. Barilla et al. This is an open access article distributed under the Creative Commons Attribution License, which permits unrestricted use, distribution, and reproduction in any medium, provided the original work is properly cited.

\begin{abstract}
We have considered a multiobjective semi-infinite programming problem with a feasible set defined by inequality constraints. First we studied a Fritz-John type necessary condition. Then, we introduced two constraint qualifications and derive the weak and strong Karush-Kuhn-Tucker (KKT in brief) types necessary conditions for an efficient solution of the considered problem. Finally an extension of a Caristi-Ferrara-Stefanescu result for the $(\Phi, \rho)$-invexity is proved, and some sufficient conditions are presented under this weak assumption. All results are given in terms of Clark subdifferential.
\end{abstract}

\section{Preliminaries and Introduction}

First, we briefly overview some notions of convex analysis and nonsmooth analysis widely used in the formulations and proofs of the main results of the paper. For more details, discussion, and applications, see [1-3].

Given a nonempty set $A \subseteq \mathbb{R}^{n}$, we denote with $\bar{A}$, $\operatorname{ri}(A)$, $\operatorname{conv}(A)$, and cone $(A)$ the closure of $A$, the relative interior of $A$, convex hull, and convex cone (containing the origin) generated by $A$, respectively. The polar cone and strict polar cone of $A$ are defined, respectively, by

$$
\begin{aligned}
A^{-} & :=\left\{x \in \mathbb{R}^{n} \mid\langle x, a\rangle \leq 0, \forall a \in A\right\}, \\
A^{s} & :=\left\{x \in \mathbb{R}^{n} \mid\langle x, a\rangle<0, \forall a \in A\right\} .
\end{aligned}
$$

It is easy to show that if $A^{s} \neq \phi$ then $\overline{A^{s}}=A^{-}$. The bipolar theorem states that

$$
A^{--}=\overline{\operatorname{cone}}(A):=\overline{\text { cone }(A)} \text {. }
$$

The cone of feasible direction of $A$ at $\hat{x} \in A$ is the cone defined by

$$
D(A, \widehat{x}):=\left\{v \in \mathbb{R}^{n} \mid \exists \delta>0, \widehat{x}+\varepsilon v \in A \forall \varepsilon \in(0, \delta)\right\} .
$$

It is worth observing that if $\hat{x}$ is a minimizer of convex function $\phi$ on a convex set $C$, then

$$
0 \in \partial \phi(\widehat{x})+N(C, \widehat{x})
$$

where $N(C, \widehat{x})$ and $\partial \phi(\widehat{x})$ denote, respectively, the normal cone of $C$ at $\hat{x}$ and the convex subdifferential of $\phi$ at $\hat{x}$; that is,

$$
\begin{aligned}
& N(C, \widehat{x}):=\left\{y \in \mathbb{R}^{n} \mid\langle y, x-\widehat{x}\rangle \leq 0 \forall x \in C\right\}, \\
& \partial \phi(\widehat{x}) \\
& \quad:=\left\{\xi \in \mathbb{R}^{n} \mid \phi(x) \geq \phi(\widehat{x})+\langle\xi, x-\widehat{x}\rangle \quad \forall x \in \mathbb{R}^{n}\right\} .
\end{aligned}
$$

We observe that if $K \subseteq \mathbb{R}^{n}$ is an arbitrary set, and $\hat{x} \in K$, then

$$
N\left(K^{-}, \widehat{x}\right)=K^{--} .
$$

If $\left\{A_{\alpha} \mid \alpha \in \Lambda\right\}$ is a collection of convex sets in $\mathbb{R}^{n}$, and $B:=\bigcup_{\alpha \in \Lambda} A_{\alpha}$, then it is easy to see that

$$
\begin{aligned}
& \operatorname{conv}(B)=\left\{\sum_{j=1}^{k} \lambda_{\alpha_{j}} a_{\alpha_{j}} \mid a_{\alpha_{j}} \in A_{\alpha_{j}}, k \in \mathbb{N}, \lambda_{\alpha_{j}}\right. \\
& \left.\geq 0, \sum_{j=1}^{k} \lambda_{\alpha_{j}}=1\right\}, \\
& \text { cone }(B)=\left\{\sum_{j=1}^{k} \lambda_{\alpha_{j}} a_{\alpha_{j}} \mid a_{\alpha_{j}} \in A_{\alpha_{j}}, k \in \mathbb{N}, \lambda_{\alpha_{j}} \geq 0\right\} .
\end{aligned}
$$


Let $\hat{x} \in \mathbb{R}^{n}$ and let $\varphi: \mathbb{R}^{n} \rightarrow \mathbb{R}$ be a locally Lipschitz function. The Clarke directional derivative of $\varphi$ at $\hat{x}$ in the direction $v \in \mathbb{R}^{n}$ and the Clarke subdifferential of $\varphi$ at $\hat{x}$ are, respectively, given by

$$
\begin{aligned}
\varphi^{0}(\widehat{x} ; v) & :=\limsup _{y \rightarrow \widehat{x}, t \downarrow 0} \frac{\varphi(y+t v)-\varphi(y)}{t}, \\
\partial_{c} \varphi(\widehat{x}) & :=\left\{\xi \in \mathbb{R}^{n} \mid\langle\xi, v\rangle \leq \varphi^{0}(\widehat{x} ; v) \quad \forall v \in \mathbb{R}^{n}\right\} .
\end{aligned}
$$

The Clarke subdifferential is a natural generalization of the classical derivative, since it is known that when function $\varphi$ is continuously differentiable at $\widehat{x}, \partial_{c} \varphi(\widehat{x})=\{\nabla \varphi(\widehat{x})\}$. Moreover when a function $\varphi$ is convex, the Clarke subdifferential coincides with the subdifferential in the sense of convex analysis.

In the following theorem we summarize some important properties of the Clarke directional derivative and the Clarke subdifferential from [1] which are widely used in what follows.

Theorem 1. Let $\varphi$ and $\phi$ be functions from $\mathbb{R}^{n}$ to $\mathbb{R}$ which are Lipschitz near $\widehat{x}$. Then,

(i) the function $v \rightarrow \varphi^{0}(\hat{x} ; v)$ is finite, positively homogeneous, and subadditive on $\mathbb{R}^{n}$, and

$$
\begin{aligned}
\varphi^{0}(\widehat{x} ; v) & =\max \left\{\langle\xi, v\rangle \mid \xi \in \partial_{c} \varphi(\widehat{x})\right\}, \\
\partial\left(\varphi^{0}(\widehat{x} ; \cdot)\right)(0) & =\partial_{c} \varphi(\widehat{x}),
\end{aligned}
$$

(ii) $\partial_{c} \varphi(\widehat{x})$ is a nonempty, convex, and compact subset of $\mathbb{R}^{n}$,

(iii) $\varphi^{0}(x ; v)$ is upper semicontinuous as a function of $(x, v)$.

In this paper, we have considered the following multiobjective semi-infinite programming problem:

$$
\begin{aligned}
\text { (MOSIP) } & \inf \left(f_{1}(x), f_{2}(x), \ldots, f_{p}(x)\right) \\
\text { s.t. } & g_{t}(x) \leq 0 \quad t \in T, \\
& x \in \mathbb{R}^{n},
\end{aligned}
$$

where $f_{i}, i \in I:=\{1,2, \ldots, p\}$ and $g_{t}, t \in T$, are locally Lipschitz functions from $\mathbb{R}^{n}$ to $\mathbb{R} \cup\{+\infty\}$ and the index set $T$ is arbitrary, not necessarily finite (but nonempty).

For differentiable MOSIP where $T$ is finite, necessary conditions of KKT type have been established under various constraint qualifications in [4]. The Abadie constraint qualification and related constraint qualification for semiinfinite systems of convex inequalities and linear inequalities are also studied in [5]. There, the characterizations of various constraint qualifications in terms of upper semicontinuity of certain multifunctions are given.

There are only a few works available that deal with optimality conditions for MOSIP. For instance, for differentiable MOSIPs, some optimality conditions have been presented by Caristi et al. in [6]. Glover et al. in [7] considered a nondifferentiable convex MOSIP and presented optimality theorems for it. For a nonsmooth MOSIP, the "basic constraint qualification" has been studied by Chuong and Kim in [8], who have given optimality and duality conditions of Karush-KuhnTucker (KKT, briefly) type for the problem which involves the notion of Mordukhovich subdifferential. Also, Gao presented some sufficient and duality results for MOSIPs under the various generalized convexity assumptions in $[9,10]$.

This paper is structured as follows: In Section 2 we propose a Fritz-John type necessary condition after we derive a KKT type necessary condition for optimality of the considered problem under a suitable qualification condition, and we establish the strong KKT necessary conditions for an efficient solution of the considered problem. In Section 3 we obtain an extension of a Caristi-Ferrara-Stefanescu result for the $(\Phi, \rho)$ invexity.

\section{Necessary Conditions}

As a starting point of this section, we denote with $M$ the feasible region of (MOSIP); that is,

$$
M:=\left\{x \in \mathbb{R}^{n} \mid g_{t}(x) \leq 0 \forall t \in T\right\} .
$$

For a given $\widehat{x} \in M$, let $T(\widehat{x})$ denote the index set of all active constraints at $\hat{x}$ :

$$
T(\widehat{x}):=\left\{t \in T \mid g_{t}(\widehat{x})=0\right\} .
$$

A feasible point $\hat{x}$ is said to be an efficient solution [resp., weakly efficient solution] to problem (MOSIP) iff there is no $x \in M$ satisfying $f_{i}(x) \leq f_{i}(\widehat{x}), i \in I$, and $\left(f_{1}(x), \ldots\right.$, $\left.f_{p}(x)\right) \neq\left(f_{1}(\widehat{x}), \ldots, f_{p}(\widehat{x})\right)$ [resp., $\left.f_{i}(x)<f_{i}(\widehat{x}), i \in I\right]$.

For each $\hat{x} \in M$, set

$$
\begin{aligned}
F_{\widehat{x}} & :=\bigcup_{i \in I} \partial_{c} f_{i}(\hat{x}), \\
G_{\widehat{x}} & :=\bigcup_{t \in T(\widehat{x})} \partial_{c} g_{t}(\hat{x}) .
\end{aligned}
$$

For each $x \in M$, set

$$
\Psi(x):=\sup _{t \in T} g_{t}(x) .
$$

Recall the following definition from [11].

Definition 2. We say that MOSIP has the Pshenichnyi-LevinValadire (PLV) property at $x \in M$, if $\Psi(\cdot)$ is Lipschitz around $x$, and

$$
\partial_{c} \Psi(x) \subseteq \operatorname{conv}\left(\bigcup_{t \in T(x)} \partial_{c} g_{t}(x)\right)=\operatorname{conv}\left(G_{x}\right) .
$$

The following condition is well known, even in differentiable cases (see, e.g., $[5,6]$ ).

Assumption $A$. The index set $T$ is a nonempty compact subset of $\mathbb{R}^{l}$, the function $(x, t) \rightarrow g_{t}(x)$ is upper semicontinuous on $\mathbb{R}^{n} \times T$, and $\partial_{c} g_{t}(x)$ is an upper semicontinuous mapping in $t$ for each $x$. 
The following lemma from [5, Theorem 5] will be used in sequel.

Lemma 3. Suppose that Assumption A holds. Then,

(1) $G_{\widehat{x}}$ is a compact set for each $\widehat{x} \in M$,

(2) the PLV property holds at each $\hat{x} \in M$.

The following result is an extension of [6, Theorem 4].

Theorem 4 (FJ necessary condition). Let $\hat{x}$ be a weakly efficient solution of MOSIP. If condition A holds at $\hat{x}$, then there exist $\alpha_{i} \geq 0$ (for $\left.i=1,2, \ldots, p\right)$ and $\beta_{t} \geq 0$, for $t \in T(\widehat{x})$, with $\beta_{t} \neq 0$ for finitely many indexes, such that

$$
\begin{aligned}
0 & \in \sum_{i=1}^{p} \alpha_{i} \partial_{c} f_{i}(\widehat{x})+\sum_{t \in T(\widehat{x})} \beta_{t} \partial_{c} g_{t}(\widehat{x}), \\
\sum_{i=1}^{p} \alpha_{i}+\sum_{t \in T(\widehat{x})} \beta_{t} & =1 .
\end{aligned}
$$

Proof. We know from Lemma 3 that $G_{\widehat{x}}$ is a compact set. Thus $F_{\widehat{x}} \cup G_{\widehat{x}}$ is also a compact set, and hence, $\operatorname{conv}\left(F_{\widehat{x}} \cup G_{\widehat{x}}\right)$ is closed.

If $0 \notin \operatorname{conv}\left(F_{\widehat{x}} \cup G_{\widehat{x}}\right)$, by strict separation theorem we find $q \in \mathbb{R}^{n}$ such that $\langle q, u\rangle<0$ for all $u \in \operatorname{conv}\left(F_{\widehat{x}} \cup G_{\hat{x}}\right)$. This implies that

$$
q \in\left(\operatorname{conv}\left(F_{\widehat{x}} \cup G_{\widehat{x}}\right)\right)^{s}=\left(F_{\widehat{x}} \cup G_{\widehat{x}}\right)^{s}=F_{\widehat{x}}^{s} \cap G_{\widehat{x}}^{s} .
$$

Since $d \in G_{\widehat{x}}^{s}$ and the PLV property is satisfied at $\hat{x}$ (by Lemma 3), we have

$$
\begin{aligned}
d & =\left(\operatorname{conv}\left(G_{\widehat{x}}\right)\right)^{s} \subseteq\left(\partial_{c} \Psi(\widehat{x})\right)^{s} \Longrightarrow \\
\Psi^{0}(\widehat{x} ; d) & <0 .
\end{aligned}
$$

Thus, there exists $\delta>0$ such that $\Psi(\widehat{x}+\varepsilon d)-\Psi(\widehat{x})<0$ for all $\varepsilon \in(0, \delta)$. The last inequality and the fact that $\Psi(\widehat{x}) \leq 0$ (since $\hat{x} \in M)$ conclude that $\Psi(\widehat{x}+\varepsilon d)<0$, and hence

$$
g_{t}(\widehat{x}+\varepsilon d)<0, \quad \forall \varepsilon \in(0, \delta) .
$$

Moreover, we have

$$
\begin{aligned}
& d \in F_{\widehat{x}}^{s}=\left(\bigcup_{i=1}^{p} \partial_{c} f_{i}(\widehat{x})\right)^{s}=\bigcap_{i=1}^{p}\left(\partial_{c} f_{i}(\widehat{x})\right)^{s} \Longrightarrow \\
& f_{i}^{0}(\widehat{x} ; d)<0 \quad \forall i=1, \ldots, p .
\end{aligned}
$$

For each $i=1, \ldots, p$ we find $\delta_{i}>0$ such that

$$
f_{i}(\widehat{x}+\varepsilon d)-f_{i}(\widehat{x})<0, \quad \forall \varepsilon \in\left(0, \delta_{i}\right)
$$

Take $\widehat{\delta}:=\min \left\{\delta, \delta_{1}, \ldots, \delta_{p}\right\}$. By (21) and (23) for each $\varepsilon \in$ $(0, \widehat{\delta})$ we have

$$
\begin{aligned}
& \left(f_{1}(\widehat{x}+\varepsilon d), \ldots, f_{p}(\widehat{x}+\varepsilon d)\right) \\
& \quad<\left(f_{1}(\widehat{x}), \ldots, f_{p}(\widehat{x})\right), \quad \widehat{x}+\varepsilon d \in M,
\end{aligned}
$$

which contradicts the weak efficiency of $\hat{x}$. This contradiction implies that

$$
0 \in \operatorname{conv}\left(F_{\widehat{x}} \cup G_{\hat{x}}\right) .
$$

Now, (7) proves the result.

The necessary conditions of Fritz-John type can be viewed as being degenerate when the multiplier corresponding to the objective function vanishes, because the function being minimized is not involved. In the next theorem we derive a Karush-Kuhn-Tuker type necessary condition for optimality of MOSIP under a suitable qualification condition.

Definition 5. Let $\hat{x} \in M$. We say that MOSIP satisfies the Zangwill CQ (ZCQ briefly) at $\hat{x}$, if

$$
G_{\widehat{x}}^{-} \subseteq \overline{D(M, \widehat{x})} .
$$

Theorem 6. Let $\hat{x}$ be a weakly efficient solution of MOSIP, ZCQ hold at $\hat{x}$, and cone $(G(\hat{x}))$ be a closed cone. Then there exist $\alpha_{i} \geq 0$ (for $\left.i=1,2, \ldots, p\right)$ and $\beta_{t} \geq 0$, for $t \in T(\widehat{x})$, with $\beta_{t} \neq 0$ for finitely many indexes, such that

$$
\begin{aligned}
0 & \in \sum_{i=1}^{p} \alpha_{i} \partial_{c} f_{i}(\widehat{x})+\sum_{t \in T(\widehat{x})} \beta_{t} \partial_{c} g_{t}(\widehat{x}) \\
\sum_{i=1}^{p} \alpha_{i} & =1
\end{aligned}
$$

Proof. We first claim that

$$
\max _{1 \leq i \leq p} f_{i}^{0}(\widehat{x} ; d) \geq 0, \quad \forall d \in D(M, \widehat{x}) .
$$

On the contrary, suppose that there exists $d \in D(M, \widehat{x})$ such that $f_{i}^{0}(\hat{x} ; d)<0$ for all $i=1, \ldots, p$. Thus, there exist positive scalars $\delta, \delta_{1}, \ldots, \delta_{p}$ such that

$$
\begin{aligned}
\widehat{x}+\varepsilon d \in M & \forall \varepsilon \in(0, \delta), \\
f_{i}(\widehat{x}+\varepsilon d)-f_{i}(\widehat{x})<0 & \forall \varepsilon \in\left(0, \delta_{i}\right) .
\end{aligned}
$$

Take $\widehat{\delta}:=\min \left\{\delta, \delta_{1}, \ldots, \delta_{p}\right\}$. By above inequalities, for each $\varepsilon \in(0, \widehat{\delta})$ we have

$$
\begin{aligned}
& \left(f_{1}(\widehat{x}+\varepsilon d), \ldots, f_{p}(\widehat{x}+\varepsilon d)\right) \\
& \quad<\left(f_{1}(\widehat{x}), \ldots, f_{p}(\widehat{x})\right), \quad \widehat{x}+\varepsilon d \in M,
\end{aligned}
$$

which contradicts the weak efficiency of $\hat{x}$. Thus, (28) is true.

If $\widehat{d} \in \overline{D(M, \widehat{x})}$, there exists a sequence $\left\{d_{k}\right\}_{k=1}^{\infty}$ in $D(M, \widehat{x})$ converging to $\widehat{d}$. Owing to (28) and continuity of function $\varphi(d):=\max _{1 \leq i \leq p} f_{i}^{0}(\widehat{x} ; d)$, we deduce that

$$
\varphi(\widehat{d})=\lim _{k \rightarrow \infty} \varphi\left(d_{k}\right) \geq 0 .
$$

We thus proved that (by assumption of ZCQ at $\widehat{x}$ )

$$
\varphi(d)=\max _{1 \leq i \leq p} f_{i}^{0}(\widehat{x} ; d) \geq 0, \quad \forall d \in G_{\widehat{x}} .
$$


Since $0 \in G_{\hat{x}}^{-}$and $\varphi(0)=0$, the last relation implies that the following convex problem has a minimum at $\bar{d}:=0$ :

$$
\begin{aligned}
\min & \varphi(d), \\
\text { subject to } & d \in G_{\widehat{x}} .
\end{aligned}
$$

Hence, by (4), (6), and (11) we obtain that

$$
\begin{aligned}
0 & \in \partial \varphi(0)+N\left(G_{\widehat{x}}^{-}, 0\right) \\
& =\operatorname{conv}\left(\bigcup_{i=1}^{p} \partial f_{i}^{0}(\widehat{x} ; \cdot)(0)\right)+G_{\widehat{x}}^{--} \\
& =\operatorname{conv}\left(F_{\widehat{x}}\right)+\overline{\operatorname{cone}}\left(G_{\widehat{x}}\right) .
\end{aligned}
$$

Now, the closeness of cone $(G(\widehat{x})),(2),(7)$, and (8) prove the results.

In almost all examples, we were not able to obtain positive KKT multipliers associated with the vector-valued objective function; that is to say, some of the multipliers may be equal to zero. This means that the components of the vectorvalued objective function do not have a role in the necessary conditions for weak efficiency. In order to avoid the case where some of the KKT multipliers associated with the objective function vanish for the MOSIP, an approach has been developed in [5], and strong KKT necessary optimality conditions have been obtained. We say that strong KKT condition holds for a multiobjective optimization problem, when the KKT multipliers are positive for all the components of the objective function. Below, we establish the necessary strong KKT conditions for an efficient solution (not a weak efficient solution) of MOSIP under a suitable qualification condition.

Let $\widehat{x} \in S$. On the lines of [4], for each $i \in I$, define the set

$$
\begin{aligned}
& Q^{i}(\widehat{x}):=\left\{x \in M \mid f_{l}(x) \leq f_{l}(\widehat{x}) \forall l \in I \backslash\{i\}\right\}, \\
& Q^{i}(\widehat{x}):=M, \quad \text { if } p=1 .
\end{aligned}
$$

For the sake of simplicity, we denote $Q^{i}(\widehat{x})$ by $Q^{i}$ in this paper.

Definition 7. Let $\hat{x} \in M$. We say that MOSIP satisfies the strong Zangwill CQ (SZCQ briefly) at $\hat{x}$, if

$$
G_{\widehat{x}}^{-} \subseteq \bigcap_{i=1}^{p} \overline{D\left(Q^{i}, \widehat{x}\right)}
$$

Theorem 8 (strong KKT necessary condition). Let $\hat{x}$ be an efficient solution of MOSIP. If in addition, SZCQ and the condition

$$
(\mathfrak{U}): \quad\left(\bigcup_{i=1}^{p} \partial_{c} f_{i}(\widehat{x})\right)^{-} \backslash\{0\} \subseteq \bigcup_{i=i}^{p}\left(\partial_{c} f_{i}(\widehat{x})\right)^{s}
$$

hold at $\hat{x}$, then $\alpha_{i}>0$ exist (for $i=1,2, \ldots, p$ ) and $\beta_{t} \geq 0$, for $t \in T(\widehat{x})$, with $\beta_{t} \neq 0$ for finitely many indexes, such that

$$
0 \in \sum_{i=1}^{p} \alpha_{i} \partial_{c} f_{i}(\widehat{x})+\sum_{t \in T(\widehat{x})} \beta_{t} \partial_{c} g_{t}(\widehat{x})
$$

Proof. We present the proof in four steps.

Step 1. We claim that

$$
\left(\bigcup_{i=1}^{p}\left(\partial_{c} f_{i}(\widehat{x})\right)^{s}\right) \cap\left(\bigcap_{i=1}^{p} D\left(Q^{i}, \widehat{x}\right)\right)=\emptyset .
$$

It suffices only to prove that

$$
\left(\partial_{c} f_{l}(\widehat{x})\right)^{s} \cap D\left(Q^{l}, \widehat{x}\right)=\emptyset \quad \forall l \in I .
$$

On the contrary, suppose that for some $l \in I$ there is a vector $d$ such that

$$
d \in\left(\partial_{c} f_{l}(\widehat{x})\right)^{s} \cap D\left(Q^{l}, \widehat{x}\right)
$$

By the definition of $D\left(Q^{l}, \widehat{x}\right)$, there exists $\delta>0$ such that $\widehat{x}+$ $\varepsilon d \in Q^{l}$ for each $\varepsilon \in(0, \delta)$. Thus, owing to the definition of $Q^{l}$ we obtain that

$$
\begin{aligned}
f_{i}(\widehat{x}+\varepsilon d) & \leq f_{i}(\widehat{x}) \quad \forall i \in I \backslash\{l\}, \forall \varepsilon \in(0, \delta), \\
\widehat{x}+\varepsilon d & \in M \quad \forall \varepsilon \in(0, \delta) .
\end{aligned}
$$

On the other hand, (41) leads to $f_{l}^{0}(\widehat{x} ; d)<0$. This means that $\delta_{l}>0$ exists, satisfying

$$
f_{l}(\widehat{x}+\varepsilon d)-f_{l}(\widehat{x})<0 \quad \forall \varepsilon \in\left(0, \delta_{l}\right)
$$

The above inequality with (42) implies that, for each $\varepsilon \in(0, \widehat{\delta})$ with $\widehat{\delta}:=\min \left\{\delta, \delta_{l}\right\}$, we have

$$
\begin{aligned}
f_{i}(\widehat{x}+\varepsilon d) & \leq f_{i}(\widehat{x}) \quad \forall i \in I \backslash\{l\}, \\
f_{l}(\widehat{x}+\varepsilon d) & <f_{l}(\widehat{x}), \\
\widehat{x}+\varepsilon d & \in M .
\end{aligned}
$$

This contradicts the efficiency of $\hat{x}$. Therefore, our claim holds.

Step 2. Let $\widehat{d} \in \overline{D\left(Q^{l}, \widehat{x}\right)}$ for some $l \in I$. Then, there exists a sequence $\left\{d_{k}\right\}_{k=1}^{\infty}$ in $D\left(Q^{l}, \widehat{x}\right)$ converging to $\widehat{d}$. By (40) and continuity of $f_{l}^{0}\left(\hat{x}_{;} \cdot\right)$ we concluded that

$$
f_{l}^{0}(\widehat{x} ; \widehat{d})=\lim _{k \rightarrow \infty} f_{l}^{0}\left(\widehat{x} ; d_{k}\right) \geq 0 .
$$

Therefore,

$$
\left(\partial_{c} f_{l}(\widehat{x})\right)^{s} \cap \overline{D\left(Q^{l}, \widehat{x}\right)}=\emptyset \quad \forall l \in I,
$$

and hence

$$
\left(\bigcup_{i=1}^{p}\left(\partial_{c} f_{i}(\widehat{x})\right)^{s}\right) \cap\left(\bigcap_{i=1}^{p} \overline{D\left(Q^{i}, \widehat{x}\right)}\right)=\emptyset .
$$

Step 3. We claim that

$$
0 \in \operatorname{ri}\left(\operatorname{conv}\left(F_{\widehat{x}}\right)\right)+\operatorname{cone}\left(G_{\widehat{x}}\right) .
$$


On the contrary we suppose that (48) does not hold. Then

$$
\operatorname{ri}\left(\operatorname{conv}\left(F_{\widehat{x}}\right)\right) \cap\left(-\operatorname{cone}\left(G_{\hat{x}}\right)\right)=\emptyset .
$$

Thus, by the strong convex separation theorem [3, Theorem 11.3 ] and noting that $\left(-\operatorname{cone}\left(G_{\widehat{x}}\right)\right)$ is a convex cone, it follows that there is a hyperplane

$$
H:=\left\{x \mid \exists d \in \mathbb{R}^{n} \backslash\{0\} \text { s.t. }\langle x, d\rangle=0\right\}
$$

separating $\operatorname{conv}\left(F_{\widehat{x}}\right)$ and $\left(-\operatorname{cone}\left(G_{\widehat{x}}\right)\right)$ properly. Hence, there exists a vector $d \in \mathbb{R}^{n}$ satisfying

$$
0 \neq d \in\left(\operatorname{conv}\left(F_{\widehat{x}}\right)\right)^{-} \cap\left(\operatorname{cone}\left(G_{\widehat{x}}\right)\right)^{-}=F_{\widehat{x}}^{-} \cap G_{\widehat{x}}^{-} .
$$

Thus, owing to SZCQ and $\mathfrak{A}$ we conclude that

$$
d \in\left(\bigcup_{i=1}^{p}\left(\partial_{c} f_{i}(\widehat{x})\right)^{s}\right) \cap\left(\bigcap_{i=1}^{p} \overline{D\left(Q^{i}, \widehat{x}\right)}\right),
$$

which contradicts (47).

Step 4. The result is immediate from (48), (8), and the fact that (see, [3, Theorem 6.9])

$$
\begin{aligned}
& \operatorname{ri}\left(\operatorname{conv}\left(F_{\widehat{x}}\right)\right) \\
& \quad \subseteq\left\{\sum_{i=1}^{p} \alpha_{i} \xi_{i} \mid \xi_{i} \in \partial_{c} f_{i}(\widehat{x}), \alpha_{i}>0, \sum_{i=1}^{p} \alpha_{i}=1\right\} .
\end{aligned}
$$

\section{Sufficient Conditions}

Similar to [6], let $\Phi: \mathbb{R}^{n} \times \mathbb{R}^{n} \times \mathbb{R}^{n+1} \rightarrow \mathbb{R}$ and $\rho: \mathbb{R}^{n} \times \mathbb{R}^{n} \rightarrow$ $\mathbb{R}$ be given functions satisfying

$$
\Phi\left(x, x^{*},(0, r)\right) \geq 0 \quad \forall x \in \mathbb{R}^{n}, r \geq 0 .
$$

Observe that an element of $\mathbb{R}^{n+1}$ is represented as the order pair $(y, r)$ with $y \in \mathbb{R}^{n}$ and $r \in \mathbb{R}$.

In [6] a differentiable function $h: \mathbb{R}^{n} \rightarrow \mathbb{R}$ was named $(\Phi, \rho)$-invex at $x^{*}$ with respect to $\mathbb{A} \subseteq \mathbb{R}^{n}$ if, for each $x \in \mathbb{A}$,

$$
\begin{gathered}
\Phi\left(x, x^{*},\left(\nabla h\left(x^{*}\right), \rho\left(x, x^{*}\right)\right)\right) \leq h(x)-h\left(x^{*}\right), \\
\Phi\left(x, x^{*}, \cdot\right) \text { is convex on } \mathbb{R}^{n+1} .
\end{gathered}
$$

We extend this result as follows.

Definition 9. The locally Lipschitz function $\hbar: \mathbb{R}^{n} \rightarrow \mathbb{R}$, at $x^{*} \in \mathbb{R}^{n}$, is called generalized $(\Phi, \rho)$-invex at $x^{*}$ with respect to $\mathbb{A} \subseteq \mathbb{R}^{n}$ if, for each $x \in \mathbb{A}$, it satisfies

$$
\begin{gathered}
\Phi\left(x, x^{*},\left(\xi, \rho\left(x, x^{*}\right)\right)\right) \leq \hbar(x)-\hbar\left(x^{*}\right) \\
\forall \xi \in \partial_{c} \hbar\left(x^{*}\right), \\
\Phi\left(x, x^{*}, \cdot\right) \text { is convex on } \mathbb{R}^{n+1} .
\end{gathered}
$$

In the rest of this paper, we will always assume $\mathbb{A}$ to be equal to the set $M$ of the feasible solution of MOSIP.
Theorem 10 (sufficient KKT condition). Suppose that there exist a feasible solution $\hat{x} \in M$ and scalars $\alpha_{i} \geq 0($ for $i \in I)$ with $\sum_{i=1}^{p} \alpha_{i}=1$ and a finite set $T^{*}:=\left\{t_{1}, t_{2}, \ldots, t_{m}\right\} \subseteq T(\widehat{x})$ and scalars $\beta_{j_{s}} \geq 0$ (for $s \in\{1,2, \ldots, m\}$ ) such that

$$
0 \in \sum_{i=1}^{p} \alpha_{i} \partial_{c} f_{i}(\hat{x})+\sum_{s=1}^{m} \beta_{t_{s}} \partial_{c} g_{t_{s}}(\widehat{x}) .
$$

Moreover if the $f_{i}$ functions and the $g_{t}$ functions $($ for $(i, t) \epsilon$ $I \times T(\hat{x}))$ are $(\Phi, \rho)$-invex at $\hat{x}$, and $\sum_{i=1}^{p} \alpha_{i} \rho_{i}(x, \hat{x})+$ $\sum_{s=1}^{m} \beta_{t_{s}} \rho_{t_{s}}(x, \hat{x}) \geq 0$ for all $x \in M$, then $\hat{x}$ is a weak efficient solution for MOSIP.

Proof. Inclusion (57) implies that some $\xi_{i} \in \partial_{c} f_{i}(\widehat{x})$ (for $i \in I$ ) and $\zeta_{t_{s}} \in \partial_{c} g_{t_{s}}(\widehat{x})$ (for $s \in\{1, \ldots, m\}$ ) exist, satisfying

$$
\begin{aligned}
\sum_{i=1}^{p} \alpha_{i} \xi_{i}+\sum_{s=1}^{m} \beta_{t_{s}} \zeta_{t_{s}} & =0, \\
\sum_{i=1}^{p} \alpha_{i}+\sum_{s=1}^{m} \beta_{t_{s}} & =b>0 .
\end{aligned}
$$

Taking $\widehat{\alpha}_{i}:=\alpha_{i} / b$ and $\widehat{\beta}_{t_{s}}:=\beta_{t_{s}} / b$, we conclude that

$$
\begin{aligned}
\sum_{i=1}^{p} \widehat{\alpha}_{i} \xi_{i}+\sum_{s=1}^{m} \widehat{\beta}_{t_{s}} \zeta_{t_{s}} & =0, \\
\sum_{i=1}^{p} \widehat{\alpha}_{i}+\sum_{s=1}^{m} \widehat{\beta}_{t_{s}} & =1 .
\end{aligned}
$$

Owing to these equalities, (54), $\sum_{i=1}^{p} \alpha_{i} \rho_{i}(x, \widehat{x})+\sum_{s=1}^{m} \beta_{t_{s}} \rho_{t_{s}}(x$, $\hat{x}) \geq 0$, and convexity of $\Phi\left(x, x^{*}, \cdot\right)$, we obtain that

$$
\begin{aligned}
0 \leq & \Phi\left(x, \widehat{x}, \sum_{i=1}^{p} \widehat{\alpha}_{i} \xi_{i}+\sum_{s=1}^{m} \widehat{\beta}_{t_{s}} \zeta_{t_{s}}, \sum_{i=1}^{p} \alpha_{i} \rho_{i}(x, \widehat{x})\right. \\
& \left.+\sum_{s=1}^{m} \beta_{t_{s}} \rho_{t_{s}}(x, \widehat{x})\right)=\Phi\left(x, \widehat{x}, \sum_{i=1}^{p} \widehat{\alpha}_{i}\left(\xi_{i}, \rho_{i}(x, \widehat{x})\right)\right. \\
& \left.+\sum_{s=1}^{m} \widehat{\beta}_{t_{s}}\left(\zeta_{t_{s}}, \rho_{t_{s}}(x, \widehat{x})\right)\right) \\
& \leq \sum_{i=1}^{p} \widehat{\alpha}_{i} \Phi\left(x, \widehat{x}, \xi_{i}, \rho(x, \widehat{x})\right) \\
& +\sum_{s=1}^{m} \widehat{\beta}_{t_{s}} \Phi\left(x, \widehat{x}, \zeta_{t_{s}}, \rho_{t_{s}}(x, \widehat{x})\right) .
\end{aligned}
$$

Now, if $\widehat{x}$ is not a weak efficient of MOSIP, there exists a point $x \in M$ such that $f_{i}(x)<f_{i}(\hat{x})$ for all $i \in I$. Hence, by generalized $(\Phi, \rho)$-invexity of $f_{i}$ functions we have

$$
\Phi\left(x, \widehat{x}, \xi_{i}, \rho_{i}(x, \widehat{x})\right) \leq f_{i}(x)-f_{i}(\widehat{x})<0 .
$$

Similarly, for each $s=1, \ldots, m$, we have

$$
\begin{aligned}
& \Phi\left(x, \widehat{x}, \zeta_{t_{s}}, \rho_{t_{s}}(x, \widehat{x})\right) \leq g_{t_{s}}(x)-g_{t_{s}}(\widehat{x})=g_{t_{s}}(x) \\
& \quad \leq 0 .
\end{aligned}
$$


From (61), (62), and $\sum_{i=1}^{p} \widehat{\alpha}_{i}>0$, we conclude that

$$
\begin{aligned}
\sum_{i=1}^{p} \widehat{\alpha}_{i} \Phi & \left(x, \widehat{x}, \xi_{i}, \rho(x, \widehat{x})\right) \\
& +\sum_{s=1}^{m} \widehat{\beta}_{t_{s}} \Phi\left(x, \widehat{x}, \zeta_{t_{s}}, \rho_{t_{s}}(x, \widehat{x})\right)<0,
\end{aligned}
$$

which contradicts (60). This contradiction shows that $\widehat{x}$ is an weak efficient for MOSIP.

Strengthening the assumptions concerning $\alpha_{i}$ s we obtain sufficient conditions for efficiency.

Theorem 11 (strong sufficient KKT condition). Suppose that there exist a feasible solution $\widehat{x} \in M$ and scalars $\alpha_{i}>0$ (for $i \in I)$ and a finite set $T^{*}:=\left\{t_{1}, t_{2}, \ldots, t_{m}\right\} \subseteq T(\widehat{x})$ and scalars $\beta_{j_{s}} \geq 0$ (for $\left.s \in\{1,2, \ldots, m\}\right)$ such that

$$
0 \in \sum_{i=1}^{p} \alpha_{i} \partial_{c} f_{i}(\widehat{x})+\sum_{s=1}^{m} \beta_{t_{s}} \partial_{c} g_{t_{s}}(\widehat{x})
$$

Moreover if the $f_{i}$ functions and the $g_{t}$ functions (for $(i, t) \in$ $I \times T(\hat{x}))$ are $(\Phi, \rho)$-invex at $\hat{x}$, and $\sum_{i=1}^{p} \alpha_{i} \rho_{i}(x, \hat{x})+$ $\sum_{s=1}^{m} \beta_{t_{s}} \rho_{t_{s}}(x, \hat{x}) \geq 0$ for all $x \in M$, then $\hat{x}$ is an efficient solution for MOSIP.

Proof. Similar to (61) and (62), if $\hat{x}$ is not efficient, we find $x \in M$ and $l \in I$ such that

$$
\begin{aligned}
\Phi\left(x, \widehat{x}, \xi_{i}, \rho_{i}(x, \widehat{x})\right) & \leq 0 \quad \forall i \in I \backslash\{l\}, \\
\Phi\left(x, \widehat{x}, \xi_{l}, \rho_{l}(x, \widehat{x})\right) & <0, \\
\Phi\left(x, \hat{x}, \zeta_{t_{s}}, \rho_{t_{s}}(x, \hat{x})\right) & \leq 0 \quad \forall s \in\{1, \ldots, m\} .
\end{aligned}
$$

From these and $\widehat{\alpha}_{i}>0$ (for all $i \in I$ ) and $\widehat{\beta}_{t_{s}} \geq 0$ (for all $s \in\{1, \ldots, m\})$, we obtain

$$
\begin{aligned}
\sum_{i=1}^{p} \widehat{\alpha}_{i} \Phi & \left(x, \widehat{x}, \xi_{i}, \rho(x, \widehat{x})\right) \\
& +\sum_{s=1}^{m} \widehat{\beta}_{t_{s}} \Phi\left(x, \widehat{x}, \zeta_{t_{s}}, \rho_{t_{s}}(x, \hat{x})\right)<0,
\end{aligned}
$$

which contradicts (60).

Remark 12. Similar to [6], we can define some weaker $(\Phi, \rho)$ invexiy assumption for the function $\hbar$, and then, we can prove some weaker sufficient conditions for optimality of MOSIP. Since the proof of these extensions is similar to previous theorems, we omit them.

\section{Competing Interests}

The authors declare that they have no competing interests.

\section{References}

[1] F. H. Clarke, Optimization and Nonsmooth Analysis, Canadian Mathematical Society Series of Monographs and Advanced Texts, John Wiley \& Sons, 1983.

[2] J. B. Hiriart-Urruty and C. Lemarechal, Convex Analysis and Minimization Algorithms, I \& II. Springer, Berlin, Germany, 1991.

[3] R. T. Rockafellar, Convex Analysis, Princeton University Press, Princeton, NJ, USA, 1970.

[4] T. Maeda, "Constraint qualifications in multiobjective optimization problems: differentiable case," Journal of Optimization Theory and Applications, vol. 80, no. 3, pp. 483-500, 1994.

[5] N. Kanzi, "On strong KKT optimality conditions for multiobjective semi-infinite programming problems with lipschitzian data," Optimization Letters, vol. 9, no. 6, pp. 1121-1129, 2015.

[6] G. Caristi, M. Ferrara, and A. Stefanescu, "Semi-infinite multiobjective programming with grneralized invexity," Mathematical Reports, vol. 62, pp. 217-233, 2010.

[7] B. M. Glover, V. Jeyakumar, and A. M. Rubinov, "Dual conditions characterizing optimality for convex multi-objective programs," Mathematical Programming, vol. 84, no. 1, pp. 201217, 1999.

[8] T. D. Chuong and D. S. Kim, "Nonsmooth semi-infinite multiobjective optimization problems," Journal of Optimization Theory and Applications, vol. 160, no. 3, pp. 748-762, 2014.

[9] X. Y. Gao, "Necessary optimality and duality for multiobjective semi-infinite programming," Journal of Theoretical and Applied Information Technology, vol. 46, no. 1, pp. 347-354, 2012.

[10] X. Y. Gao, "Optimality and duality for non-smooth multiple objective semi-infinite programming," Journal of Networks, vol. 8, no. 2, pp. 413-420, 2013.

[11] N. Kanzi, "Constraint qualifications in semi-infinite systems and their applications in nonsmooth semi-infinite problems with mixed constraints," SIAM Journal on Optimization, vol. 24, no. 2, pp. 559-572, 2014. 


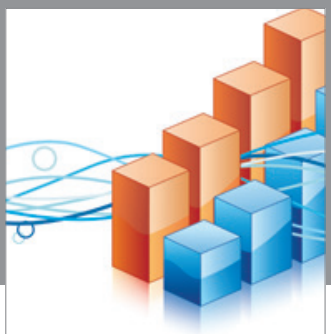

Advances in

Operations Research

vatem alat4

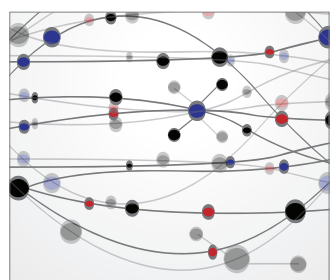

\section{The Scientific} World Journal
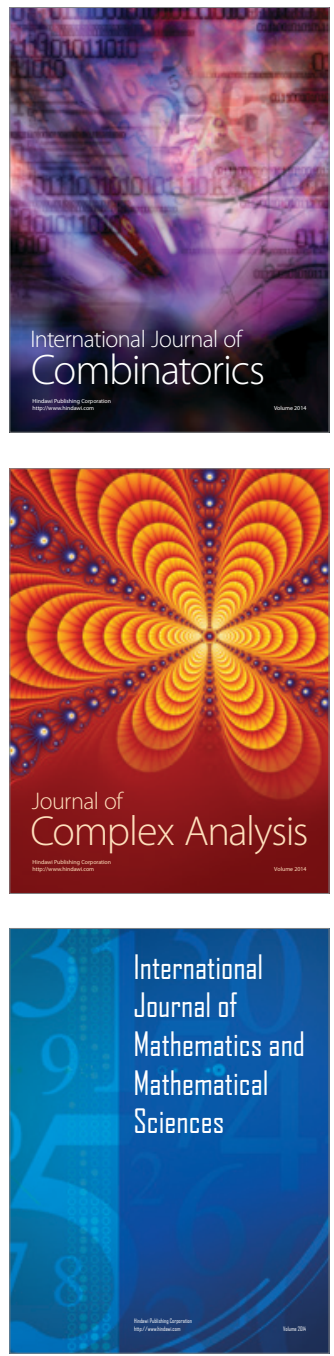
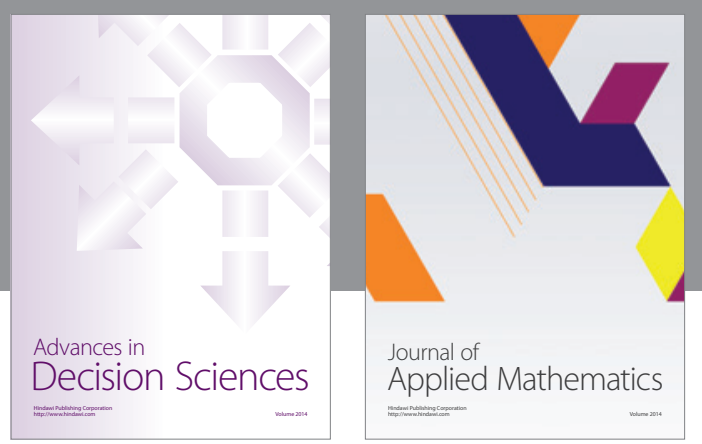

Algebra

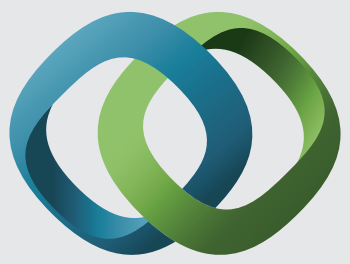

\section{Hindawi}

Submit your manuscripts at

http://www.hindawi.com
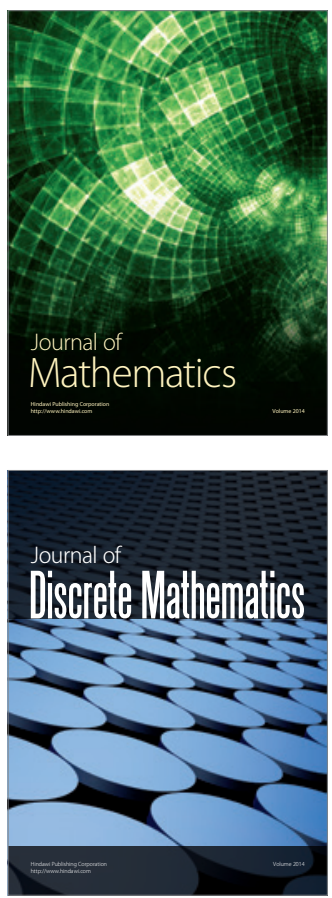

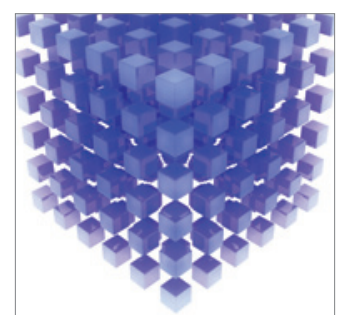

Mathematical Problems in Engineering
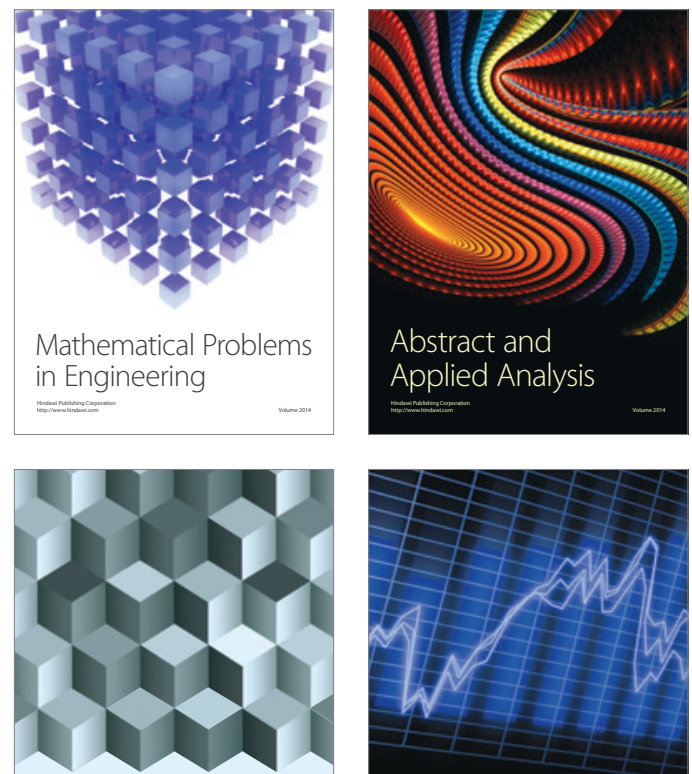

Journal of

Function Spaces

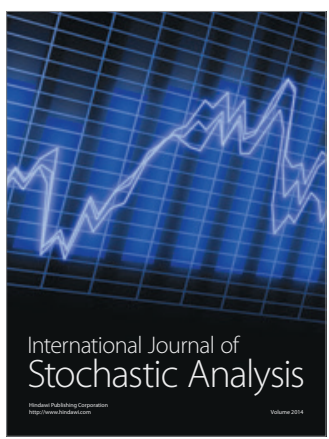

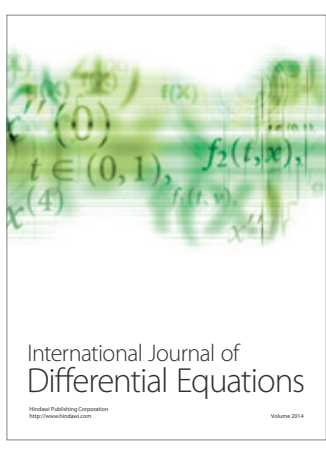
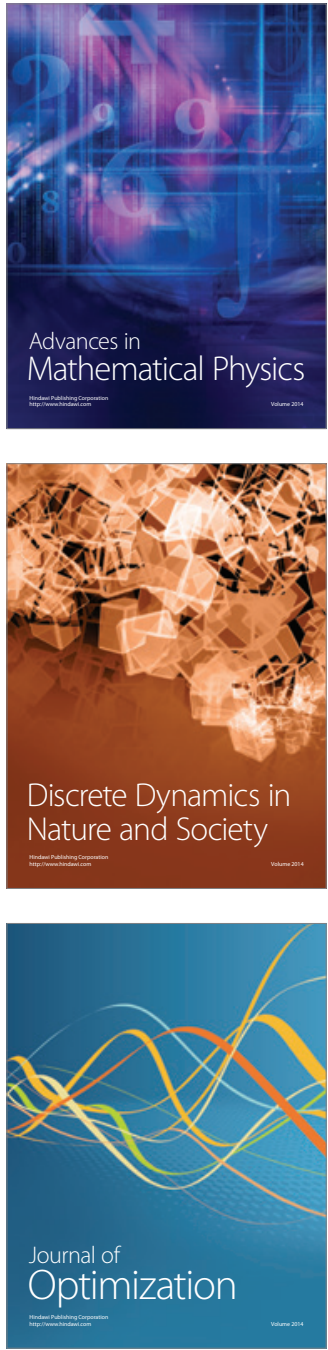